\title{
Opioid Prescribing and Opioid Risk Mitigation Strategies in the Veterans Health Administration
}

\author{
Friedhelm Sandbrink, $M D^{1,2,3,4}{ }^{\oplus}$, Elizabeth M. Oliva, PhD ${ }^{5,6}$, Tara L. McMullen, $P h D^{7}$, \\ Amy R. Aylor, PharmD, MS ', Michael A. Harvey, PharmD', \\ Melissa L. Christopher, PharmD ${ }^{7}$, Francesca Cunningham, PharmD', \\ Taeko Minegishi, MS ${ }^{8,9}$, Thomas Emmendorfer, PharmD ${ }^{7}$, and Jenie M. Perry, BS ${ }^{7}$
}

\begin{abstract}
'Pain Management and Opioid Safety Program, Veterans Health Administration, Washington, DC, USA; ${ }^{2}$ Department of Neurology, Washington DC VA Medical Center, Washington, DC, USA; ${ }^{3}$ Uniformed Services University, Bethesda, MD, USA; ${ }^{4}$ George Washington University, Washington, DC, USA; ${ }^{5}$ VA Program Evaluation and Resource Center, VA Office of Mental Health and Suicide Prevention, Menlo Park, CA, USA; ${ }^{V}$ VA Center for Innovation to Implementation, VA Palo Alto Health Care System, Menlo Park, CA, USA; ${ }^{7}$ Patient Care Services, Pharmacy Benefits Management Service, Veterans Health Administration, Washington, DC, USA; ${ }^{8}$ Partnered Evidence-based Policy Resource Center, VA Boston Healthcare System, Boston, MA, USA; ${ }^{9}$ Bouvé College of Health Sciences, Northeastern University, Boston, MA, USA.
\end{abstract}

INTRODUCTION: The Veterans Health Administration (VHA) has taken a multifaceted approach to addressing opioid safety and promoting system-wide opioid stewardship.

AIM: To provide a comprehensive evaluation of current opioid prescribing practices and implementation of risk mitigation strategies in VHA.

SETTING: VHA is the largest integrated health care system in the United States.

PROGRAM DESCRIPTION: VHA prescribing data in conjunction with implementation of opioid risk mitigation strategies are routinely tracked and reviewed by VHA's Pharmacy Benefits Management Services (including Academic Detailing Service) and the Pain Management Program Office. Additional data are derived from the Partnered Evidence-Based Policy Resource Center (PEPReC) and from a 2019 survey of interdisciplinary pain management teams at VHA facilities. Prescribing data are reported quarterly until first quarter fiscal year 2020 (Q1FY2020), ending December 31, 2019.

PROGRAM EVALUATION: VHA opioid dispensing peaked in 2012 with 679,376 Veterans receiving an opioid prescription, and when including tramadol, in 2013 with 869,956 Veterans. Since 2012, the number of Veterans dispensed an opioid decreased 56\% and co-prescribed opioid/benzodiazepine decreased $83 \%$. Veterans with high-dose opioids ( $\geq 100 \mathrm{mg}$ morphine equivalent daily dose) decreased 77\%. In Q1FY2020, among Veterans on long-term opioid therapy (LTOT), 91.1\% had written informed consent, $90.8 \%$ had a urine drug screen, and 89.0\% had a prescription drug monitoring program query. Naloxone was issued to 217,469 Veterans and resulted

Prior Presentations The data presented are derived from clinical dashboards that are routinely reported for internal communication within the VHA. Selected quality improvement data from the Opioid Safety Initiative were previously reported at the American Academy of Pain Medicine (March 17, 2017).

Electronic supplementary material The online version of this article (https://doi.org/10.1007/s11606-020-06258-3) contains supplementary material, which is available to authorized users.

Received February 9, 2020

Accepted September 18, 2020

Published online November 16, 2020 in $>1,000$ documented overdose reversals. In 2019, interdisciplinary pain management teams were fully designated at $68 \%$, partially designated at $28 \%$, and not available at $4 \%$ of 140 VA parent facilities. Fifty percent of Veterans on opioids at very high risk for overdose/suicide received interdisciplinary team reviews.

IMPLICATIONS: VHA clinicians have greatly reduced their volume of opioid prescribing for pain management and expanded implementation of opioid risk mitigation strategies.

IMPACTS: VHA's integrated health care system provides a model for opioid stewardship and interdisciplinary pain care.

KEY WORDS: VHA; opioids; pain management; opioid epidemic; academic detailing.

J Gen Intern Med 35(Suppl 3):S927-S34

DOI: $10.1007 /$ s11606-020-06258-3

(c) Society of General Internal Medicine (This is a U.S. government work and not under copyright protection in the U.S.; foreign copyright protection may apply) 2020

\section{INTRODUCTION}

Chronic pain is more common and severe in Veterans than in non-Veterans. ${ }^{1}$ It is estimated that one in three Veterans receiving primary care from a Veterans Health Administration (VHA) facility has a chronic pain diagnosis and one in ten Veterans has persistent, severe pain. ${ }^{2}$ The most common pain conditions in Veterans are musculoskeletal including joint, lower back, and neck pain. ${ }^{1}$ Chronic pain in Veterans is often complicated by concurrent mental health disorders such as posttraumatic stress disorder and substance use disorder. ${ }^{3,4}$

In the late 1990s and early 2000s, clinicians in the United States (US) increasingly incorporated opioid medication into care plans for their patients with severe pain, and VHA providers followed the national trend. By 2010, opioid prescribing was recognized as an important contributor to the US opioid crisis. ${ }^{5}$ Studies in 2011 and 2019, respectively, showed that VHA patients had nearly twice the risk of fatal accidental 
poisoning compared with the general US population ${ }^{4}$ and that among Veterans receiving opioid prescriptions for pain, higher opioid doses were associated with increased risk of opioid overdose death. ${ }^{6}$ In response, VHA began to develop standardized metrics for opioid use, and after successful piloting in several facilities in 2012, the Opioid Safety Initiative (OSI) was launched system-wide in 2013 . $^{7}$

VHA employed four broad strategies to address the opioid epidemic: education, pain management, risk mitigation, and addiction treatment. ${ }^{7}$ This evaluation focuses on the first three strategies. Foundational to this effort is ongoing monitoring of key OSI-related measures with VHA dashboards making opioid dispensing data and implementation of risk mitigation strategies visible to providers and administrators. A key element of VHA's OSI education strategy is VHA Pharmacy Benefits Management's Academic Detailing Service (PBM ADS). Academic Detailing (AD) is a knowledge translation intervention through educational outreach delivered by clinicians to providers and staff with the goal of aligning their practice with current evidence. ${ }^{8-10}$ In VHA, this is primarily done in one-on-one, face-to-face settings by specially trained clinical pharmacists who use OSI dashboards with prescriberlevel data and educational tools to inform and engage clinicians in adopting best practices ${ }^{11}$ (see OSI toolkit on VHA Pain Management Program Office's website ${ }^{12}$ ). Regarding OSI patient education, since 2014, VHA policy mandated educating all Veterans exposed to long-term opioid therapy (LTOT) and obtaining written informed consent. ${ }^{13}$

Implementation of OSI risk mitigation strategies expanded greatly with the 2016 Comprehensive Addiction and Recovery Act (CARA) ${ }^{14}$ that mandated enhancements to opioid safety and pain care in VHA including expanded monitoring of opioid prescribing and risk mitigation. In 2016, the Centers for Disease Control and Prevention (CDC) issued their guideline for prescribing opioids for chronic pain ${ }^{15}$ and this was followed in 2017 by the VA/DoD Clinical Practice Guideline (CPG) for Opioid Therapy for Chronic Pain. ${ }^{16}$ Notably, the VA/DoD CPG specifically recommended against initiation of LTOT for chronic pain. In 2016, policy mandated querying state prescription drug monitoring programs (PDMPs) for patients receiving a controlled substance prescription for longer than 5 days (except for hospice care).$^{17}$ To further enhance risk mitigation, VHA policy since 2018 has required interdisciplinary team review and care coordination for Veterans estimated to be at very high risk for overdose/suicide based on the Stratification Tool for Opioid Risk Mitigation (STORM) which utilizes predictive analytics to estimate Veterans' risk for overdose or suicide. ${ }^{18}$

The OSI pain management strategy consists of expanding pain care treatment options and making those available system-wide and readily accessible for Veterans. Access to cognitive behavioral therapy for chronic pain, already available at many VHA facilities since the 1990s, was expanded further. CARA legislation from 2016 required full implementation of the Stepped Care Model for Pain Management, expanded access to Complementary and Integrative Health (CIH) modalities including designated flagship sites, and pain management teams (PMTs) at all VHA facilities. Also in 2016, VHA's State of the Art (SOTA) conference on nonpharmacological approaches to chronic musculoskeletal pain identified non-pharmacological therapies with sufficient evidence for VHA-wide implementation. ${ }^{19}$ In compliance with CARA and incorporating SOTA recommendations, in 2017, VHA mandated interdisciplinary PMTs at all facilities and specified that they include medical providers with pain management expertise and offer evidence-based behavioral therapy approaches, physical medicine and rehabilitation therapies, and evaluation and treatment of opioid use disorder (OUD); concurrently, a separate Whole Health initiative has mandated access to $\mathrm{CIH}$ modalities. The VA Maintaining Internal Systems and Strengthening Integrated Outside Networks (MISSION) Act of $2018^{20}$ greatly expanded access to nonVHA providers and subsequently, VHA replaced the Veterans Choice program in June 2019 with the VA Community Care Network.

Monitoring implementation and compliance with the wide range of legislation and policies to improve pain care and opioid safety has relied heavily on data analytics that leverage VHA's electronic health record (EHR) system. This evaluation will describe opioid safety-related data routinely reviewed by VHA program offices and survey data related to implementation of interdisciplinary pain management teams across VHA.

\section{METHODS}

This evaluation was conducted by VHA's Pharmacy Benefits Management Services (PBM) and National Pain Program Office as part of a large-scale national Quality Improvement project. Data related to interdisciplinary team reviews of veryhigh-risk STORM patients were part of a national program evaluation ${ }^{21}$ with a study protocol approved by the VA Boston Institutional Review Board (IRB 3069; R\&D\#3244-X).

\section{Data Sources}

Corporate Data Warehouse. PBM compiled most of the data in this study from VHA's Corporate Data Warehouse ${ }^{22}$ (CDW), a repository of national VHA administrative data. Opioid, benzodiazepine, and laboratory data were extracted from CDW from the beginning of Quarter 4 Fiscal Year 2012 to the end of Quarter 1 Fiscal Year 2020 (Q4FY2012Q1FY2020; July 1, 2012-December 31, 2019).

Other Sources. A customer relationship management platform from Academic Detailing Services (ADS) tracked opioid-safety-related educational outreach visits from Q1FY2015 to Q1FY2020. Spontaneous reported opioid overdose reversals with outpatient naloxone were reported to the Opioid Overdose Education and Naloxone Distribution 
$(\mathrm{OEND})^{7,11}$ from May 2014 through February 2016 and then captured with national VHA EHR notes from October 31, 2017, to December 31, 2019. The VHA Internal Audit Office surveyed VHA parent facilities between February and March 2019 to assess compliance with VHA mandates regarding interdisciplinary PMT implementation. A VHA national randomized program evaluation identified very-high-risk STORM patients ${ }^{23}$ who received interdisciplinary risk reviews from Q3FY2018 to Q1FY2020. ${ }^{24}$

Data Analysis/Measures. Data were extracted to determine measures and calculate morphine equivalent daily dosing (MEDD). Opioid measures include all opioids, with some variation in regard to tramadol, buprenorphine products, medication for opioid use disorder, and liquid injectable, and suppository forms of opioids, as noted for specific measures per definitions (see figures). Opioid dosage calculation for MEDD was calculated according to CDC surveillance methods. ${ }^{25}$

Risk Mitigation Strategies. Pharmacy Benefits Management (PBM) OSI Dashboard: Quarterly opioid dispensing data tracked and monitored - at national, regional, and facility levels - the number of Veterans: (1) dispensed opioids, (2) coprescribed opioids and benzodiazepines, (3) dispensed high-dose opioids defined as $\geq 100 \mathrm{mg}$ average MEDD, and (4) receiving LTOT with a urine drug screen within the past year. Opioid dispensing data were monitored for all groups since Q4FY2012. Since Q1FY2015, VHA also monitored VHA patients receiving opioid prescriptions from a VHA pharmacy that were prescribed by Veterans Choice Program or VA Community Care Network providers. Changes in opioid utilization and risk mitigation were calculated by determining number and percent decrease by quarter over time.

Academic Detailing Services (ADS) Measures: Quarterly ADS assessments to support opioid risk mitigation were used to track and monitor (1) patients dispensed opioids with an annual PDMP check since Q1FY2017 ${ }^{17}$ and (2) outpatient naloxone prescription measures since Q3FY2014: (2a) patients dispensed naloxone, (2b) naloxone fills, (2c) naloxone prescribers, (2d) patients co-prescribed opioids and benzodiazepines dispensed naloxone in the past year, and (2e) patients with $\geq 50 \mathrm{mg} \mathrm{MEDD}{ }^{25}$ dispensed naloxone in the past year.

Interdisciplinary Risk Reviews: Quarterly assessments of patients prescribed opioids who were estimated by STORM to be at very high risk for overdose/suicide and who had an interdisciplinary risk review documented in the EHR have been tracked since Q3FY2018. ${ }^{24}$ Change in very-high-risk patients reviewed was calculated from Q3FY2018 to Q1FY2020.

Pain Management Strategies. VHA Survey of Interdisciplinary Pain Management Teams (PMTs): A VHA Internal Audit Office survey assessed implementation of
PMTs with providers for four mandated categories-(1) medical pain management, (2) behavioral therapy, (3) rehabilitation medicine, and (4) integrated access to assessment and treatment for opioid use disorder. A fully designated PMT included named providers for all four categories and a partially designated PMT named providers in one to three categories.

Education Strategies. ADS Measures: Quarterly ADS assessments to support patient and provider education were used to track and monitor: (1) patients receiving LTOT with written informed consent ${ }^{13}$ since Q3FY2014 and (2) educational outreach academic detailer visits related to pain/OSI and OEND via a customer relationship management platform since Q1FY2015.

\section{RESULTS}

Risk Mitigation Strategies. PBM OSI Dashboard: Opioid prescribing in VHA peaked in Q4FY2012 with 679,376 patients receiving an opioid prescription, equivalent to $17.2 \%$ of Veterans with pharmacy activity that quarter. Since then, the number of Veterans receiving an opioid decreased by $56 \%$ to 297,251 patients in Q1FY2020, equivalent to 7.0\% of Veterans with pharmacy activity (Fig. 1). The graph shows a gradual decline from Q4FY2012 to Q4FY2013, followed by a steeper, essentially linear decline until Q1FY2020 (average quarterly reduction of 58,966 since Q4FY2013). Data, including tramadol, identified a peak in opioid prescribing 1 year later, in Q4FY2013 with 869,956 Veterans receiving an opioid prescription and a $57 \%$ reduction to 377,427 Veterans in Q1FY2020.

Opioid/benzodiazepine co-prescribing was reduced by $83 \%$ between Q4FY2012 and Q1FY2020 (from 122,633 to 20,266 patients; see Fig. 1), equivalent to $6.8 \%$ of Veterans dispensed opioids. Historical VHA data identified Q2FY2012 as the actual peak of opioid/benzodiazepine co-prescribing (125,478 Veterans).

High-dose opioid prescribing ( $\geq 100 \mathrm{mg}$ MEDD) decreased from 59,499 to 13,738 Veterans during this time period, a $77 \%$ reduction (Fig. 1), with historical VHA data identifying Q4FY2011 as the actual peak (60,299 Veterans).

Regarding past-year urine drug screening among patients on LTOT, there was a $148 \%$ increase between Q4FY2012 and Q1FY2020 (36.6 to 90.8\%, respectively; Fig. 2).

The number of VHA patients dispensed opioids from VHA that were prescribed by a Veterans Choice Program/VA Community Care Network provider increased greatly over time to 16,831 in Q1FY2020 (Fig. 1). Notably, during the transition from the Veterans Choice Program to the VA Community Care Network - between 2018 and 2019 - there was a marked reduction in VHA dispensing opioids prescribed by these providers. 

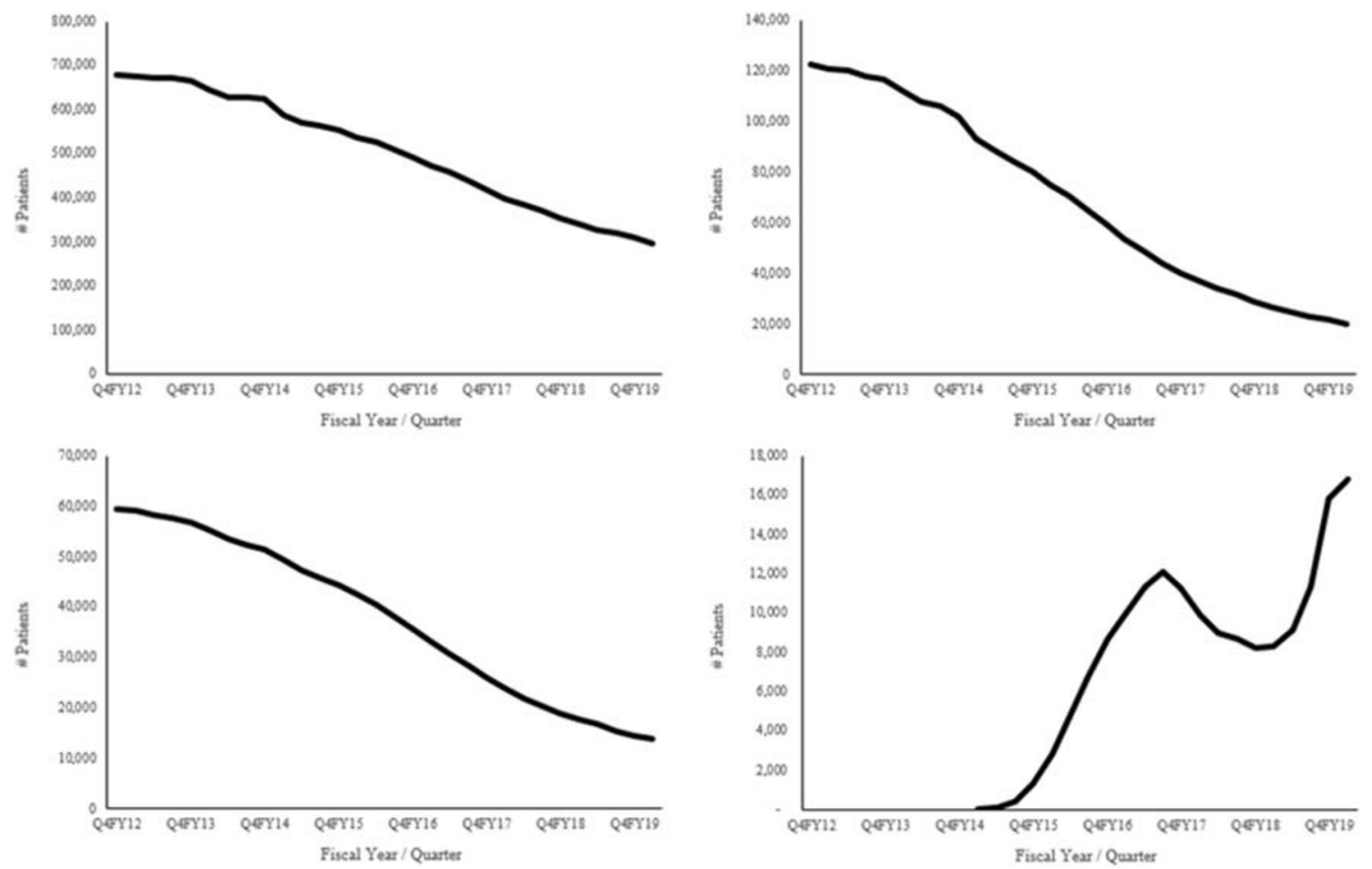

Figure 1 Quarterly opioid prescribing data. Top left panel: total number of VHA patients dispensed an opioid. PBM OSI dashboard data: opioids dispensed includes buprenorphine products, excludes tramadol (when OSI began, VHA's Drug File classified buprenorphine products

in the opioid class; for consistency in the metric across time, it has continued to be included and internal examination of inclusion of buprenorphine in this metric found that it did not significantly impact results). Top right panel: total number of VHA patients dispensed opioids and benzodiazepines. PBM OSI dashboard data: opioids include buprenorphine products (see above) and tramadol; benzodiazepines exclude clonazepam (when OSI began VHA's Drug File originally classified it as a seizure medication only and later additionally classified it as a benzodiazepine; for consistency in the metric across time it was excluded, however, internal examination of inclusion of clonazepam in this metric found that it did not significantly impact results). Bottom left panel: total number of VHA patients dispensed high-dose ( $\geq 100 \mathrm{mg}$ morphine equivalent daily dose [MEDD]) opioids. PBM OSI dashboard data: high-dose opioids include buprenorphine (see above) and tramadol. Bottom right panel: total number of VHA patients dispensed opioids from a VHA pharmacy that were prescribed by a non-VHA prescriber. PBM OSI dashboard data: opioids dispensed excludes buprenorphine/naloxone and tramadol. Data were available beginning in Q1FY2015 when tracking began (early low rates may be due to learning curves involved with uptake of new processes, including how to record the prescriptions). The Veterans Choice program transitioned to the VA Community Care Network in 2018 with a significant reduction in VHA patients dispensed opioids from a VHA pharmacy that were prescribed by a non-VHA prescriber during this transition period. Further evaluation of this reduction may be warranted, with a focus on the factors.

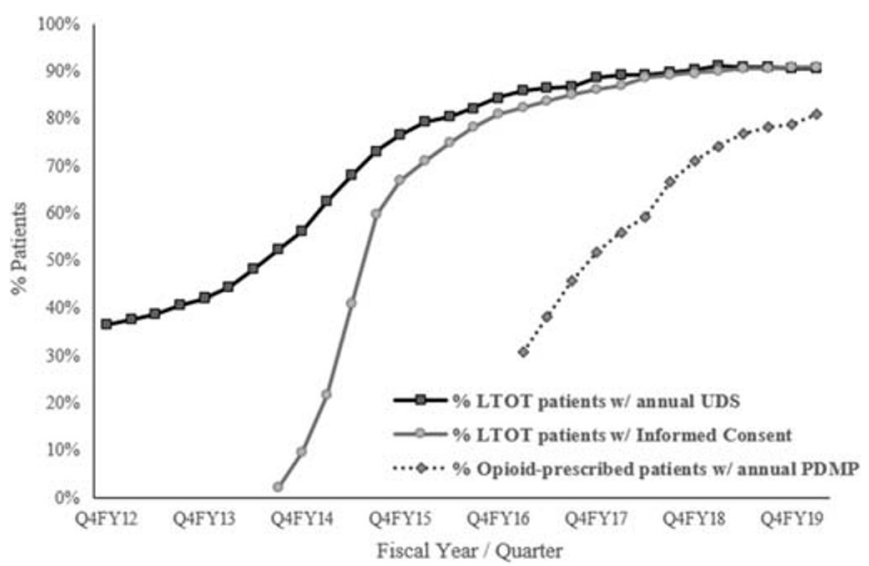

Figure 2 Quarterly opioid risk mitigation data. \% LTOT patients with annual UDS: percentage of VHA patients receiving long-term opioid therapy with a urine drug screen (UDS) in the past year. PBM OSI dashboard data: defines LTOT as $\geq 90$ days supply of opioids for the selected quarter and the prior quarter (opioids exclude buprenorphine/naloxone and tramadol). \% LTOT patients with informed consent: percentage of VHA patients receiving long-term opioid therapy with informed consent (from Q3FY2014). ADS measure: LTOT defined per VHA Directive $1005^{10}(\geq 90$ days supply of schedule II or III opioids for the selected quarter and the prior quarter; opioids exclude schedule III and IV opioids such as tramadol and codeine as well as medications for opioid use disorder such as methadone solution and sublingual buprenorphine). \% Opioid-prescribed patients with annual PDMP: percentage of VHA patients dispensed opioids with an annual Prescription Drug Monitoring Program check. ADS measure: opioids include tramadol. Per the PDMP policy issued in October 2016, excluded patients with a 5-day supply or less of opioids without refills and hospice patients. Data available since Q1FY2017. 

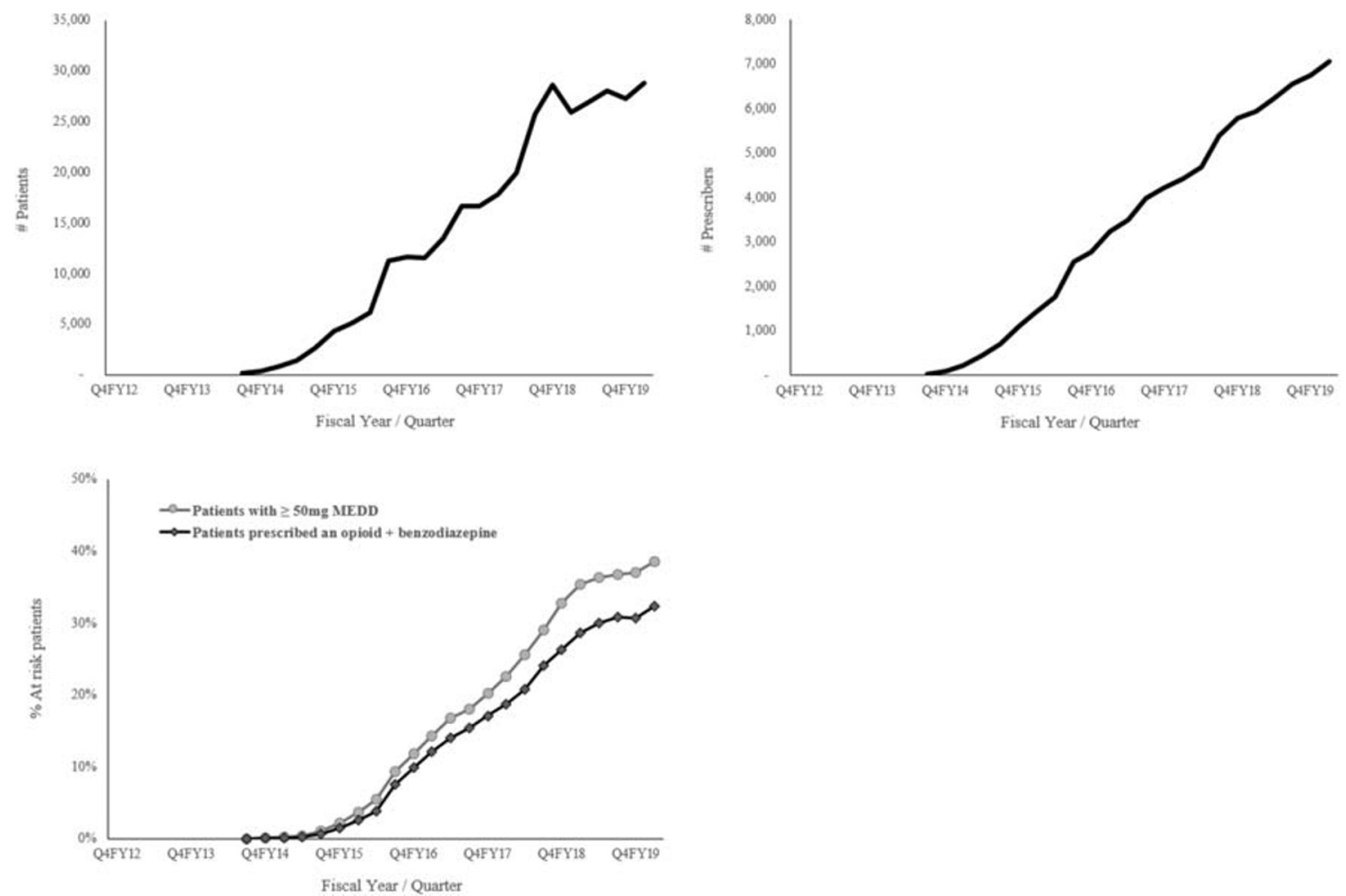

Figure 3 VHA Academic Detailing Service naloxone-related measures data (since Q3FY2014). Top left panel: total number of VHA patients dispensed outpatient naloxone. Top right panel: total number of VHA outpatient naloxone prescribers. Bottom left panel: percentage of VHA patients with $\geq 50 \mathrm{mg}$ morphine equivalent daily dose (MEDD) of opioids with a naloxone prescription in the past year and with overlapping opioid and benzodiazepine prescriptions with naloxone in the past year.

ADS Measures: PDMP query rates increased to $81.0 \%$ of all patients dispensed opioids and $89.0 \%$ of patients on LTOT in Q1FY2020 (Fig. 2). Through Q1FY2020, VHA's OEND program dispensed naloxone to 217,469 unique Veterans (Fig. 3), increasing distribution to patients with overlapping opioid and benzodiazepine prescriptions to $32.3 \%$ in Q1FY2020 as well as to $38.6 \%$ among patients with $\geq 50 \mathrm{mg}$ MEDD (Fig. 3). These efforts have results in more than 1,000 opioid overdose reversals reported between Q3FY2014 and Q1FY2020.

Interdisciplinary Risk Reviews: Between Q3FY2018 and Q1FY2020, 50.0\% of Veterans estimated by STORM to be at very high risk for overdose/suicide had a documented interdisciplinary team review $(22,250$ of 44,534 Veterans) (Fig. 4).

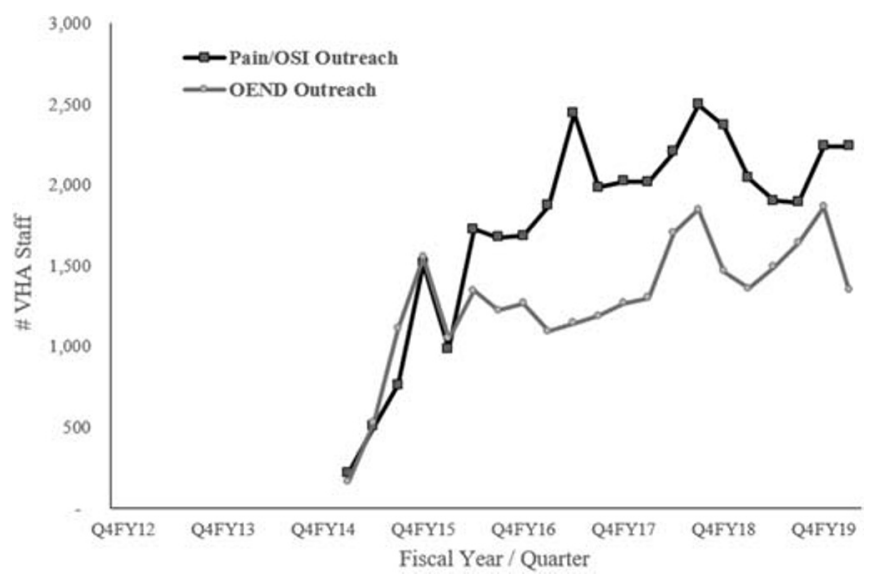

Figure 4 Stratification Tool for Opioid Risk Mitigation (STORM)-identified very high-risk patients prescribed opioids with a data-based opioid risk review data (since FY18Q3). 


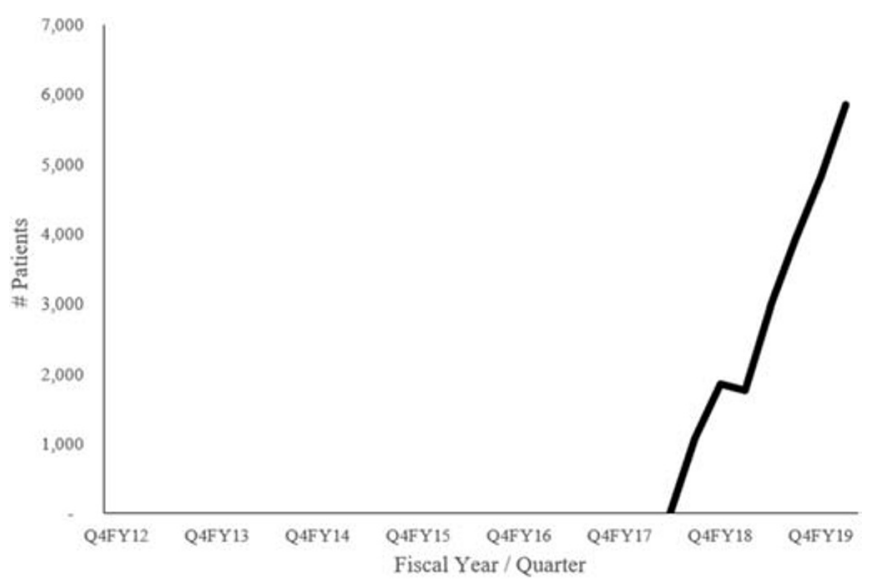

Figure 5 VHA Academic Detailing Service Opioid-Related Educational Outreach by Academic Detailers data (since Q1FY2015). Total number of VHA clinicians with a pain/Opioid Safety Initiative (OSI)-related Academic Detailing visit. Total number of VHA clinicians with an Opioid Overdose Education and Naloxone Distribution (OEND)-related Academic Detailing visit.

Pain Management Strategies. VHA Survey of Interdisciplinary PMTs: The 2019 survey found that 95 (68\%) facilities had a fully designated PMT, 29 (28\%) had a partially designated PMT, and 6 (4\%) did not have any designated PMT members. Among facilities with partial PMTs, 13 had only one or two PMT roles filled. The PMT roles not filled included 26 facilities without a provider with addiction management expertise for assessment and treatment of opioid use disorder, 21 facilities without integrated rehabilitation medicine, 15 facilities without behavioral therapy, and 14 facilities without a medical provider with pain expertise.

Educational Strategy. Informed consent for patients on LTOT steadily increased to $91.1 \%$ in Q1FY2020 (Fig. 2). Since the national rollout of AD in Q1FY2015, academic detailers have had 43,689 educational outreach visits with 22,000 unique staff related to pain/OSI or OEND (Fig. 5).

\section{DISCUSSION}

VHA implemented its Opioid Safety Initiative (OSI) nationwide in 2013 and this evaluation extends previous findings regarding the impact of the $\mathrm{OSI}^{26,27}$ by demonstrating dramatic changes in opioid prescribing, utilization of risk mitigation by VHA providers, and patient and provider education. Since 2012, opioid dispensing has steadily decreased from a peak of $17.2 \%$ of Veterans with pharmacy activity to recently $7.0 \%$. Several opioid prescribing measures were already beginning to stabilize or even reverse at that time, as providers became increasingly aware of the risks associated with opioid prescribing for chronic pain, in particular high-dose LTOT. The OSI data demonstrate a reduction in the number of Veterans receiving any opioid, a decrease in opioid dosages, and an initial shift towards tramadol prescribing and dispensing.
Specifically, high-dose opioid therapy peaked in Q4FY2011 whereas opioid dispensing excluding tramadol peaked in Q4FY2012, and total opioid dispensing that included tramadol peaked 1 year later (Q4FY2013). Remarkably, the number of Veterans with opioid therapy has decreased steadily since 2013, well in advance of the CDC Guideline for Prescribing Opioids for Chronic Pain (2016) ${ }^{15}$ and the VA/DoD Clinical Practice Guideline for Opioid Therapy for Chronic Pain (2017). ${ }^{16}$ Marked decreases in high-risk opioid prescribing are noted, namely high-dose prescribing and opioid/ benzodiazepine co-prescribing (77\% and $83 \%$ respectively). The recent slowing in quarterly reductions suggests potentially reaching a bottom in the near future for both measures.

The OSI dashboard provided transparency of provider and facility prescribing patterns across the enterprise. Quarterly updates about the metrics were provided to facility leadership and stakeholders, and facilities were asked to review the data with specific attention to Veterans on very-high opioid dosages, beginning with greater than $1000 \mathrm{mg}$ MEDD and expanding to all patients with more than $200 \mathrm{mg}$ MEDD.

These clinical metrics in combination with opioid safety focused educational initiatives contributed to awareness of clinical guidelines and risk mitigation strategies. ADS pharmacists educated and assisted providers, specifically targeting high prescribers. The marked reduction in opioid and benzodiazepine co-prescribing may also be explained by a separate initiative targeting the prescribing of psychotropic drugs including benzodiazepines. ${ }^{28}$

VHA has greatly expanded access to non-pharmacological modalities including behavioral, rehabilitation, and $\mathrm{CIH}$ approaches and patient-centric whole health ${ }^{19,29-31}$ as part of its pain care transformation. VHA's 2009 National Pain Directive established the Stepped Care Model for Pain Management, firmly rooted in the biopsychosocial model for pain assessment and treatment. The 2017 mandate of interdisciplinary PMTs across all 
facilities expands access to multimodal pain specialty care with integrated access to non-pharmacological strategies. Based on a prior internal VHA survey, 105 of 141 VHA parent facilities had a pain medicine specialty clinic or center in 2014. Many of these pain clinics, however, had largely consultative functions and did not offer integrated access to evaluation and treatment of OUD. With the 2017 mandate, Pain Clinics shifted their focus to provide oversight and actual prescribing of opioid medication and integrated access to evaluation and treatment of OUD and - consistent with VHA's whole-person approach - to reduce reliance on opioid medication.

Concerns regarding the potential unintended harm to patients discontinued or tapered rapidly from LTOT, even at previously high dosage, are important to consider when establishing guidance for providers and facility leaders. The authors of the 2017 VA/DoD Opioid Therapy CPG specifically recommended individualizing opioid tapering based on risk assessment and patient needs and characteristics, and warned against abrupt discontinuations of LTOT. Based on the CPG, the ADS opioid tapering ${ }^{32}$ tool recommends individualized care with slow tapering if risks outweigh benefits of LTOT, suggesting a $5-20 \%$ reduction per 28 days with pauses as needed, and a patient-centered approach with patient buy-in. The 2017 VA/DoD CPG and ADS tapering tool align with recently published tapering guidance and clarification released by $\mathrm{HHS}^{33,34}$ and the authors of the CDC guideline. ${ }^{15,35} \mathrm{~A}$ study of VHA prescribing data indicated that reductions in LTOT accounted for $83 \%$ of the overall decline in opioid prescription fills between 2010 and 2016 and estimated that $90 \%$ of LTOT reductions were due to declining rates in initiation of LTOT. ${ }^{27}$ These findings are encouraging as not newly starting Veterans on LTOT is specifically recommended by the VA/DoD Opioid CPG. ${ }^{16}$

Despite the VA/DoD Opioid Therapy CPG and ADS tapering tool expressing caution about opioid tapering and mandating patient-centered approaches, anecdotal evidence points to Veterans experiencing harm from having their opioid medication discontinued or tapered faster than clinically indicated or despite significant challenges with reductions. A recently published observational study showed that patients were at greater risk of death from overdose or suicide after stopping opioid treatment, with an increase in the risk the longer patients had been on opioid therapy before stopping. ${ }^{36}$

To mitigate such risks, many Veterans on opioid therapy may benefit from greater care coordination to address medical and mental health comorbidities and providers should be aware of signs of potential opioid use disorder. Our evaluation found that interdisciplinary risk reviews of very-high-risk patients is feasible. Less than 2 years since the process was mandated for all facilities, the data presented here show that half of all Veterans at very high risk have undergone such interdisciplinary care coordination reviews, and we are currently analyzing data to determine the outcomes of this risk mitigation intervention.
A recent study of drug overdose deaths in VHA patients showed a decrease in the number of Veterans dispensed a prescription opioid in the 3 months prior to an overdose (from $54 \%$ in 2010 to $26 \%$ in 2016). ${ }^{37}$ Nevertheless, overdose deaths in Veterans from opioids mirror data for the US population with a marked increase in deaths that include synthetic opioids, primarily fentanyl products. Thus, expanding access to OUD care including Medications for OUD is essential. VHA has mandated including providers with addiction medicine expertise in interdisciplinary PMTs at all facilities.

This comprehensive evaluation demonstrates that an integrated, interdisciplinary health care system approach to improve pain care and opioid safety is feasible and impactful. VHA's OSI and related system-wide initiatives have greatly reduced the volume of opioid medication for pain management, expanded implementation of opioid risk mitigation to improve safety for Veterans who receive opioid medication, and transformed pain management in VHA towards integrated, multimodal pain care, delivered collaboratively by primary care and specialty care teams.

Acknowledgments: The authors wish to acknowledge the contribution of Specialty Care Services, Pharmacy Benefits Management, and the Office of Mental Health and Suicide Prevention within the Veterans Health Administration (VHA) and VHA leadership who made this work possible by establishing dashboards for clinical care monitoring that form the basis for this report. The authors thank the VHA Internal Audit Office for providing data about the implementation of Pain Management Team at VHA facilities.

Corresponding Author: Friedhelm Sandbrink, MD; Department of Neurology, Washington DC VA Medical Center, Washington, DC, USA (e-mail: Friedhelm.Sandbrink@va.gov).

Funding The work for this evaluation was performed by the authors as part of their routine duties.

\section{Compliance with Ethical Standards:}

Conflict of Interest: The authors declare that they do not have a conflict of interest.

\section{REFERENCES}

1. Nahin RL. Severe pain in Veterans: the effect of age and sex, and comparisons with the general population. J Pain. 2017:18(3):247-254.

2. Carey E, Leonard C. Caring for Veterans Reporting Chronic Pain: Provider experiences and trends in the prevalence of chronic pain. Available at: https://www.hsrd.research.va.gov/for_researchers/cyber_ seminars/archives/video_archive.cfm?SessionID=2351. Accessed June 25, 2020.

3. Lew HL, Otis JD, Tun C, Kerns RD, Clark ME, Cifu DX. Prevalence of chronic pain, posttraumatic stress disorder, and persistent postconcussive symptoms in OIF/OEF veterans: Polytrauma clinical triad. J Rehabil Res Dev. 2009;46(6):697-702.

4. Bohnert AS, Igen MA, Galea S, McCarthy JF, Blow FC. Accidental poisoning mortality among patients in the Department of Veterans Affairs Health System. Med Care. 2011;49(4):393-6.

5. Okie S. A flood of opioids, a rising tide of deaths. $\mathrm{N}$ Engl $\mathrm{J}$ Med. 2010;363(21):1981-5.

6. Bohnert AS, Igen MA. Understanding links among opioid use, overdose, and suicide. N Engl J Med. 2019;380(1):71-9. 
7. Gellad WF, Good CB, Shulkin DJ. Addressing the opioid epidemic in the United States: lessons from the Department of Veterans Affairs. JAMA Intern Med. 2017;177(5):611-612.

8. Avorn J, Soumerai SB. Improving drug-therapy decisions through educational outreach. A randomized controlled trial of academically based “detailing.” N Engl J Med. 1983;308(24):1457-1463.

9. Wells DL, Popish S, Kay C, Torrise V, Christopher ML. VA Academic Detailing Service: Implementation and Lessons Learned. Fed Pract. 2016;33(5):66-69

10. Popish SJ. Academic detailing: Using clinical evidence to improve care. Ment Health Clin. 2013;2(12):392-394.

11. Bounthavong M, Harvey MA, Wells DL, et al. Trends in naloxone prescriptions prescribed after implementation of a National Academic Detailing Service in the Veterans Health Administration: A preliminary analysis. J Am Pharm Assoc. 2017;57:S68-72.

12. U.S. Department of Veterans Affairs. Opioid Safety Initiative. (2020). Available at: https://www.va.gov/PAINMANAGEMENT/Opioid_Safety_ Initiative_OSI.asp. Accessed June 25, 2020.

13. U.S. Department of Veterans Affairs. Informed Consent for Long-Term Opioid Therapy for Pain. VA Directive 1005. 2014. Available at: https:// www.va.gov/VHAPUBLICATIONS/ViewPublication.asp?pub_ID=8822 . Accessed June 25, 2020.

14. Comprehensive Addiction and Recovery Act of 2016, Pub. L. No 114-198, Stat. 524 (2016).

15. The Centers for Disease Control and Prevention. CDC Guideline for Prescribing Opioids for Chronic Pain- United States, 2016. MMWR. 2016; 65(1): 1-49.

16. U.S. Department of Veterans Affairs. VA/DoD Clinical Practice Guideline for Opioid Therapy for Chronic Pain. Available at: https://www.healthquality.va.gov/guidelines/Pain/cot/VADoDOTCPG022717.pdf. Accessed June 25, 2020

17. U.S. Department of Veterans Affairs. Querying State Prescription Drug Monitoring Programs (PDMP). VA Directive 1306. 2016. Available at: https://www.va.gov/vhapublications/ViewPublication.asp?pub_ ID=3283. Accessed June 25, 2020.

18. U.S. Department of Veterans Affairs. Conduct of Data-Based Case Reviews of Opioid-Exposed Patients With Risk Factors. VA Notice 201915. 2019.

19. Kligler B, Bair MJ, Banerjea R, DeBar L, Ezeji-Okoye, Lisi A, Murphy JL, Sandbrink F, Cherkin DC. Clinical Policy Recommendations From the VHA State-of-the-Art Conference on Non-Pharmacological Approaches to Chronic Musculoskeletal Pain. J Gen Intern Med 2018;33(Suppl 1):16-23

20. John S. McCain III, Daniel K. Akaka, and Samuel R. Johnson VA Maintaining Internal Systems and Strengthening Integrated Outside Networks Act of 2018 (VA MISSION Act of 2018), Pub. L. No 115-182, Stat. 2372 (2018).

21. Minegishi T, Frakt AB, Garrido MM, et al. Randomized program evaluation of the Veterans Health Administration Stratification Tool for Opioid Risk Mitigation (STORM): A research and clinical operations partnership to examine effectiveness. Subst Abus. 2019;40(1):14-19.

22. Zhang P. VIReC Data Contents: Corporate Data Warehouse (CDW), [14 Domains]. 2017 Mar 21. Available from: http://vaww.virec.research.va. gov/CDW/Documentation.htm, Accessed June 25, 2020.

23. Oliva EM, Bowe T, Tavakoli S, et al. Development and applications of the Veterans Health Administration's Stratification Tool for Opioid Risk
Mitigation (STORM) to improve opioid safety and prevent overdose and suicide. Psychol Serv . 2017;14(1):34-39.

24. Minegishi T, Garrido MM, Pizer SD, Frankt, AB. Effectiveness of policy and risk targeting for opioid-related risk mitigation: a randomized programme evaluation with stepped-wedge design. BMJ. 2018;8:e020097.

25. Department of Health and Human Services Centers for Disease Control and Prevention. Calculating total daily dose of opioids for safer dosage. Available at: https://www.cdc.gov/drugoverdose/pdf/calculating_total_ daily_dose-a.pdf. Accessed June 25, 2019.

26. Lin LA, Bohnert ASB, Kerns RD, Clay MA, Ganoczy D, Ilgen MA. Impact of the Opioid Safety Initiative on opioid-related prescribing in veterans. Pain. 2017;158(5):833-839.

27. Hadlandsmyth K, Mosher H, Vander Weg MW, Lund BC. Decline in prescription opioids attributable to decreases in long-term use: a retrospective study in the Veterans Health Administration 2010-2016. J Gen Intern Med. 2018;33:818-824.

28. Harris Ragan AP, Aikens GB, Bounthavong M, Brittain K, Mirk A. Academic Detailing to Reduce Sedative-Hypnotic Prescribing in Older Veterans. J Pharm Pract. 2019:897190019870949.

29. Frank JW, Carey E, Nolan C, Kerns RD, Sandbrink F, Gallagher R, Ho PM. Increased Nonopioid Chronic Pain Treatment in the Veterans Health Administration, 2010-2016. Pain Med. 2019; 20(5):869-877.

30. U.S. Department of Veterans Affairs. Provision of Complementary and Integrative Health (CIH). Available at: https://www.va.gov/VHAPUBLICATIONS/ViewPublication.asp?pub_ID=5401. Accessed June 25, 2020.

31. Herman PM, Yuan AH, Cefalu MS et al. The use of complementary and integrative health approaches for chronic musculoskeletal pain in younger US Veterans: An economic evaluation. PLoS One. 2019;14(6):e0217831.

32. U.S. Department of Veterans Affairs. Opioid Taper Decision Tool. Available at: https://www.pbm.va.gov/PBM/AcademicDetailingService/Documents/Academic_Detailing_Educational_Material_Catalog/52_Pain_Opioid_Taper_Tool_IB_10_939_P96820.pdf. Accessed June 25, 2020.

33. Department of Health and Human Services Centers. HHS Guide for Clinicians on the Appropriate Dosage Reduction or Discontinuation of Long-Term Opioid Analgesics. Available at: https://www.hhs.gov/ opioids/sites/default/files/2019-10/Dosage_Reduction_Discontinuation.pdf. Accessed June 25, 2020.

34. Dowell D, Compton WM, Giroir BP. Patient-Centered Reduction or Discontinuation of Long-term Opioid Analgesics. The HHS Guide for Clinicians. JAMA. 2019;322(19): 1855-1856.

35. The Centers for Disease Control and Prevention. Pocket Guide: Tapering Opioids for Chronic Pain. Available at: https://www.cdc.gov/drugoverdose/pdf/clinical_pocket_guide_tapering-a.pdf . Accessed June 25, 2020.

36. Oliva EM, Bowe T, Manhapra A, Kertesz S, Hah JM, Henderson P, Robinson A, Paik M, Sandbrink S, Gordon AJ, Trafton JA. Associations Between Stopping Prescriptions for Opioids, Length of Opioid Treatment, and Overdose or Suicide Deaths in US Veterans: Observational Evaluation BMJ 2020;368:m283. https://doi.org/10.1136/bmj. $\mathrm{m} 283$.

37. Lin LA, Peltzman T, McCarthy JF, Oliva EM, Trafton JM, Bohnert A. Changing trends in opioid overdose deaths and prescription opioid receipt among Veterans. Am J Prev Med. 2019; 57(1):106-110.

Publisher's Note: Springer Nature remains neutral with regard to jurisdictional claims in published maps and institutional affiliations. 